\title{
Integration of breast cancer gene signatures based on graph centrality
}

\author{
Jianxin Wang ${ }^{1 *+}$, Gang Chen ${ }^{1+}$, Min Li ${ }^{1,2}$, Yi Pan ${ }^{1,2^{*}}$ \\ From BIOCOMP 2010 - The 2010 International Conference on Bioinformatics and Computational Biology \\ Las Vegas, NV, USA. 12-15 July 2011
}

\begin{abstract}
Background: Various gene-expression signatures for breast cancer are available for the prediction of clinical outcome. However due to small overlap between different signatures, it is challenging to integrate existing disjoint signatures to provide a unified insight on the association between gene expression and clinical outcome.

Results: In this paper, we propose a method to integrate different breast cancer gene signatures by using graph centrality in a context-constrained protein interaction network (PIN). The context-constrained PIN for breast cancer is built by integrating complete PIN and various gene signatures reported in literatures. Then, we use graph centralities to quantify the importance of genes to breast cancer. Finally, we get reliable gene signatures that are consisted by the genes with high graph centrality. The genes which are well-known breast cancer genes, such as TP53 and BRCA1, are ranked extremely high in our results. Compared with previous results by functional enrichment analysis, graph centralities, especially the eigenvector centrality and subgraph centrality, based gene signatures are more tightly related to breast cancer. We validate these signatures on genome-wide microarray dataset and found strong association between the expression of these signature genes and pathologic parameters.

Conclusions: In summary, graph centralities provide a novel way to connect different cancer signatures and to understand the mechanism of relationship between gene expression and clinical outcome of breast cancer. Moreover, this method is not only can be used on breast cancer, but also can be used on other gene expression related diseases and drug studies.
\end{abstract}

\section{Background}

A gene signature is a group of genes whose expression pattern represents the status of a gene expression disease [1]. By using the microarray technology, which has developed rapidly in last ten years, various gene signatures are developed for various complex diseases, especially the cancer. Since researchers found that gene-expression signatures are able to predict clinical outcome of breast cancer in 2002 [2,3], it have become a hot topic and attracted the attention of both biologists and oncologists. Signatures for various phenotypes, such as poor prognosis [3], invasiveness [4],

\footnotetext{
* Correspondence: jxwang@mail.csu.edu.cn; pan@cs.gsu.edu

† Contributed equally

'School of Information Science and Engineering, Central South University, Changsha, 410083, China

Full list of author information is available at the end of the article
}

recurrence [5], and metastasis [6,7], have been experimentally derived from patient groups and biological hypotheses. However, distinct signatures share very few genes, even though they paradoxically occupy a common prognosis space. For both cancer biologists and oncologists, a critical problem is whether these disjoint genetic signatures can provide a unified insight on the relationship between gene expression and clinical outcome.

Obviously, complex heterogeneity of signatures caused by different probe design, different platforms, or inadequate patient samples, becomes an obstacle when trying to integrate various signatures of breast cancer. Gene Ontology enrichment, pathway analysis, and some genome-scale methods are proposed to explain the lack of overlap [8-10]. In literature [8], the authors list five
Ciomed Central

(c) 2011 Wang et al. This is an open access article distributed under the terms of the Creative Commons Attribution License (http:// creativecommons.org/licenses/by/2.0), which permits unrestricted use, distribution, and reproduction in any medium, provided the original work is properly cited. 
possible explanations for the small overlap between signatures:

1. Heterogeneity in expression due to different platform technologies and references;

2. Differences in supervised protocols with which signatures are extracted;

3. Although the genes are not exactly the same, they represent the same set of pathways;

4. Differences in clinical composition between datasets (i.e. sample heterogeneity);

5. Small sample size problems that cause inaccurate signatures.

Through a large-scale analysis that performed on 947 breast cancer samples from Affymetrix platform, the authors of literature [8] conclude that the small signature overlap is most likely due to small sample size problem (explanation 5). However, the conclusion might be specific to the datasets and the specific techniques used in their work. By comparison of three prognostic gene expression signatures for breast cancer, literature [9] suggested that the small overlap between the different prognostic gene signatures is because these different signatures represented largely overlapping biological processes (explanation 3). By taking into account the biological knowledge that exists among different signatures, the authors of [10] found that different signatures are similar at biological level, rather than gene level (explanation 3). Much work has been done in an effort to understand the small overlap between gene signatures, but so far there is no widely accepted explanation.

Meanwhile, computational biologists have developed Protein Interaction Networks(PIN) that effectively have been used to analyze protein interactions underpinning share sub-phenotypes among otherwise seemingly disparate disease, such as retinitis pigmentosa, epithelial ovarian cancer, inflammatory bowel disease, amyotrophic lateral sclerosis, Alzheimer disease, type 2 diabetes, coronary heart disease [11] and head and neck tumor metastasis [12]. For an individual expression signature in breast cancer, protein interaction networks are successfully used to predict prognosis [13] and detect subnetwork signatures of metastatic disease [4]. More recently, in [14], on genome-wide coexpression networks for different disease states, the authors used univariate Cox model and Relief algorithm to select the genes that are the most predictive of clinical outcome to construct gene signature for lung cancer. A 13-gene lung cancer prognosis signature with significant prognostic stratifications is identified by this method. By Single Protein Analysis of Net-works(SPAN [12]) and conservative permutation re-sampling, a small, but more biological significant breast cancer signature consisted by 54 genes is identified from a protein interaction network include 250 cancer-related genes curated from literatures $[15,16]$. In reference [10], by integrating biological knowledge and different signatures, the authors derived a unified signature that is more robust than original signatures.

However, to integrate different breast cancer signatures, most existing methods need cancer domain knowledge, such as cancer-related literature used in $[10,15,16]$. This limited the application of these methods. In this paper, we describe a method to integrate different breast cancer signatures by using graph centrality in a context-constrained PIN for human breast cancer which is constructed by integrating disjoint gene signatures reported in previous literatures. Unlike most existing methods, the method proposed in this paper is able to integrate distinct gene signatures without cancer domain knowledge. By Gene Ontology (GO) enrichment analysis, Kyoto Encyclopedia of Genes and Genomes (KEGG) enrichment analysis and relating our results to previous biological studies, we show that the genes in centrality-based signatures are tightly related to breast cancer and are able to predict clinical outcome.

\section{Methods}

To identify reliable gene signature of breast cancer by integrating various gene signatures, we propose a graph centrality based method to identify disease genes from a constrained PIN and the overview of this method is provided in Figure 1. Briefly, as shown in Figure 1, the method proposed here has three steps:

1. Collect genes from different breast cancer gene signatures, and discard the genes that exist in only one signature.

2. Project the genes collected in Step 1. to human PIN to construct a context-constrained PIN that consisted. Therefore, to some extent, all genes in this context-constrained network are related to breast cancer. However, we don't know which genes are the most important ones to the breast cancer and can be used to predict clinical outcome.

3. To determine the relationship between genes and breast cancer, we calculated graph centrality of each gene in this constrained PIN. Since the constrained PIN is built based on breast cancer gene signatures, graph centrality of genes in this network indicates their relationship to breast cancer. Output given number of genes with highest graph centrality as the new unified breast cancer signature.

Details of the three steps are described in following three subsections and then validation methods are presented. 


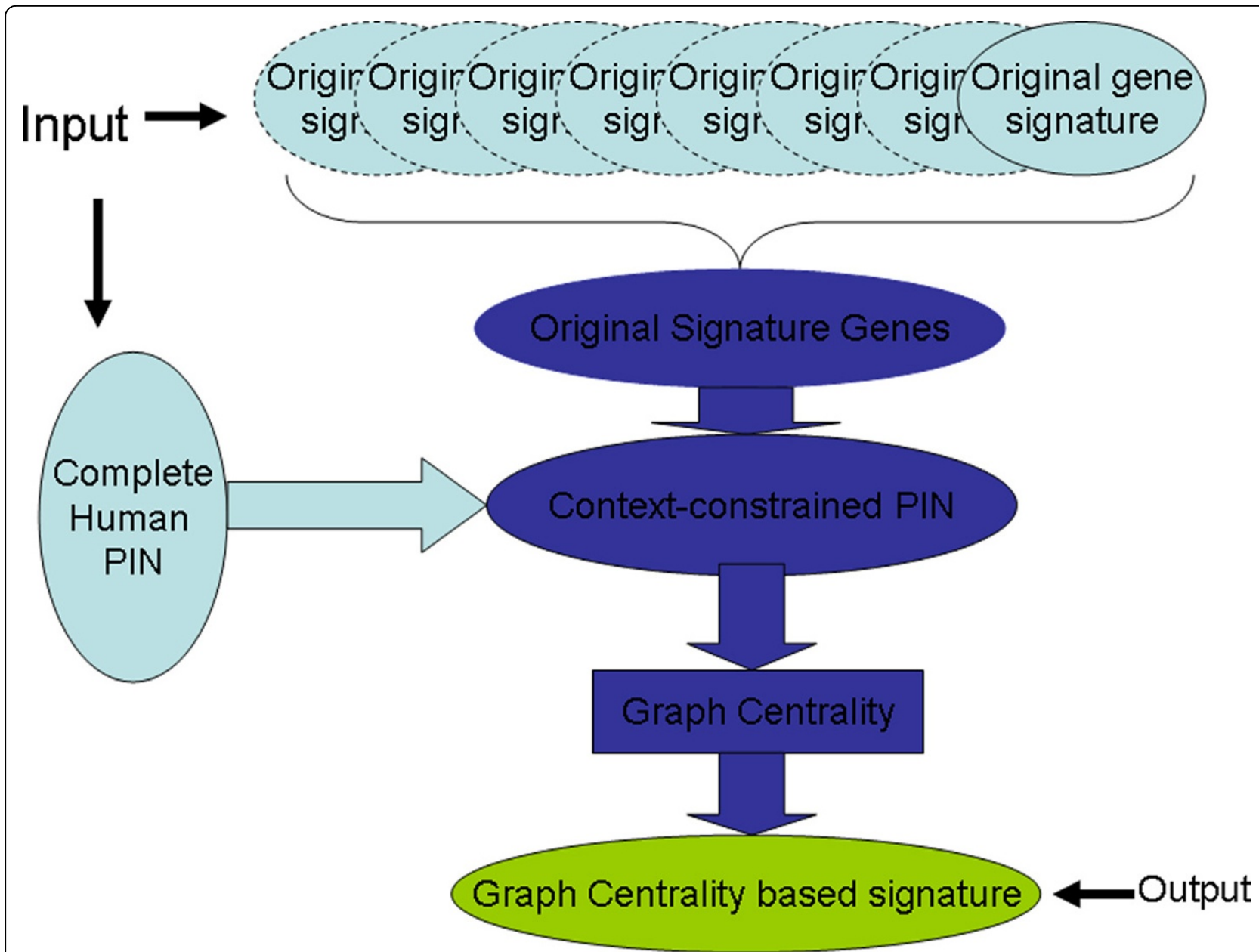

Figure 1 Schematic overview of graph centrality based integration of gene signatures. Schematic overview of graph centrality based integration of distinct breast cancer gene signatures.

\section{Collecting genes from different signatures}

GeneSignDB (http://compbio.dfci.harvard.edu/gene$\operatorname{sigdb} /)$ [17] is a curated gene signatures database that collected gene signatures for various species and diseases. Keywords "breast cancer" for disease and "human" for species are used to search gene signatures for human breast cancer in GeneSignDB. 94 distinct human breast cancer signatures are obtained, which are reported in 58 different literatures. Since the genes which are included in only one gene signature may be generated by chance, we discard these unreliable genes.

\section{Construction of context-constrained PIN}

A complete human PIN is constructed by integrating protein interaction data from Human Protein Reference Database (HPRD) and BioGRID interaction database $[18,19]$. After removal of duplicate edges and self- interactions, we got a PIN that is consisted by 51057 distinct interactions among 11465 proteins.

Then, the genes we collected in the first step are projected to the complete human PIN and a constrained PIN for human breast cancer is obtained. This constrained PIN contains 2924 proteins and 4698 interactions.

Use graph centrality to quantify the relationship between genes and breast cancer

Various definitions of graph centrality have been proposed from different perspectives to evaluate the importance of nodes in a graph. The concept has been widely used in bioinformatics, such as discovery of essential proteins in protein networks [20]. Because it is difficult to infer which definition is best for identifying disease genes in the context-constrained network, we evaluated six different definitions in our work. 
For a protein interaction network $G(V, E)$, the six measurements of centrality used in this study are defined as following:

- Degree centrality $(D C)$ : The degree centrality $D C(i)$ of vertex $i$ is the number of edges connecting node $i$ and its neighbors [21].

$\operatorname{DC}(i)=\operatorname{Deg}(i)$

where $\operatorname{Deg}(i)$ is the degree of vertexes $i$.

- Betweenness centrality $(B C)$ : The betweenness centrality $B C(i)$ of a node $i$ is the average fraction of shortest paths that pass through the node $i$ [22].

$B C(i)=\sum_{s} \sum_{t} \frac{\sigma_{s t}(i)}{\sigma_{s t}}, s \neq t \neq i$,

where $\sigma_{s t}$ denotes the total number of shortest paths between $s$ and $t$ and $\sigma_{s t}(i)$ denotes the number of shortest paths from $s$ to $t$ that pass through the node $i$.

- Closeness centrality(CC): The closeness centrality $C C$ of node $i$ can defined as [23]:

$C C(i)=\frac{1}{\sum_{j \neq i} c_{p}(i, j)}$

$C C$ is a global metric which describes how the given node $i$ connects to other nodes.

- Subgraph centrality(SC): The subgraph centrality $S C(i)$ of node $i$ can be defined as [24]:

$S C=\sum_{l=0}^{\infty} \frac{\mu_{l}(i)}{l !}$

where $\mu_{l}(i)$ denotes the number of closed walks of length $l$ which starts and ends at node $i$.

- Eigenvector centrality(EC): The eigenvector centrality $E C(i)$ of node $i$ is defined as the $i$ th component of the principal eigenvector of $A$, where $A$ is an adjacent matrix. Let $\lambda$ be an eigenvalue and $e$ be the eigenvector. Then for an equation $\lambda e=A e$, we can obtain $E C(i)=e_{1}(i)$, where $e_{1}$ corresponds to the largest eigenvalue of $A$ [25].
- Information centrality(IC): The information centrality $I C(i)$ of node $i$ in a is defined as [26]:

$$
\operatorname{IC}(i)=\left[\frac{1}{n} \sum_{j} \frac{1}{I_{i j}}\right]^{-1},
$$

where $n$ is the number of nodes in graph $G$ and $I_{i j}=$ $\left(r_{i i}+r_{j j}-r_{i j}\right)-1$, where $r_{i j}$ is the element of matrix $R$. Let $D$ be a diagonal matrix of the weighted degree of each node and $J$ be a matrix with all its elements equal to one. Then, we get $R=\left(r_{i j}\right)=[D-A+J]-1$. For computational purposes, $I_{i i}$ is defined as infinite. Thus, $\frac{1}{I_{i i}}=0$.

High centrality of a gene indicates that it is important to the constrained PIN and probably plays an important role in mechanism of breast cancer development. Therefore, according to the graph centrality of genes, we get a gene list that is ordered by the genes' importance to human breast cancer. Depending on specific purpose, a given number of top genes can be selected to construct a reliable gene signature of breast cancer. The reliable gene signature is the integration of the disjoint original signatures.

\section{KEGG pathway and Gene Ontology enrichment analysis}

$p$-value based on the hypergenometirc distribution is widely used as a measurement of the extent to which the clusters are annotated by a specific GO term [27-30]. Basically, the $p$-value is defined as following:

$$
P=1-\sum_{i=0}^{k-1} \frac{\left(\begin{array}{c}
C \\
i
\end{array}\right)\left(\begin{array}{c}
G-C \\
n-i
\end{array}\right)}{\left(\begin{array}{l}
G \\
n
\end{array}\right)}
$$

where $C$ is the size of the gene set containing $k$ gene with a given GO term; $G$ is the size of the universal set of known genes and contains $n$ genes with the annotation.

Low $P$ in Formula 6 indicates that the module closely corresponds to the GO annotation because the network has a rare chance to produce the module. To simplify our analysis, we define $p$-score as the negative of $\log (P)$ with the annotation [31].

Gene set enrichment analysis for KEGG pathways is very similar to the one for $\mathrm{GO}$ annotations. In Equation $6, C$ is the size of the gene set containing $k$ genes that exist in a given KEGG pathway; $G$ is the size of the universal set of known genes and contains $n$ genes that exist in the pathway. Similarly, $p$-score can be used to measure the relationship between the gene set and a specific KEGG pathway. 
In this study, both KEGG and GO enrichment analysis are performed on DAVID [32].

\section{Validate on microarray dataset}

To evaluate the signature's ability to predict clincal outcome, we used expression intensity of the genes in the signature to cluster microarray datasets of breast cancer patients with different pathologic parameters. Patients with similar pathologic parameters should be clustered togather. For a given pathologic parameter, the $p$-value of the clustering result indicates the signature's ability to predict the pathologic parameter.

In this study, euclidean distance between samples are calculated by using the expression intensity of genes in gene signature. Then hierarchical clustering is used to cluster the microarry datasets of breast cancer patients.

\section{Results}

\section{Overlap among breast cancer signatures}

As mentioned in Methods section, 94 breast cancer gene signatures are obtained from public database. Since the different generation methods and the various purposes of these signatures, the size of these signatures are very different. The biggest signature contains 3260 genes, and the smallest signature only contains 4 genes. The median size of these signatures is 46 .

To evaluate the similarity among these gene signatures, we analyze the overlap among the 94 gene signatures (see Table 1). The analysis result is very consistent with the results reported in literature [15]. A very small overlap is found among different signatures. 4143 (58.6\% of the total number) genes are found in only one signature, but only $24(0.4 \%)$ genes overlapped 10 or more signatures, and none of the genes overlapped all 94 signatures. The lack of overlapping is an obstacle to integrate various signatures of breast cancer.

\section{Centrality based gene signatures}

All genes included in the 94 signatures are projected to the human PIN described in Methods section. In consideration of the lower reliability of gene signatures, only the genes that are included in two or more different signatures are used to construct the context-constrained PIN of breast cancer. Finally, this contextconstrained PIN contains 2924 proteins and 4698 interactions.

Then, six graph centralities of each genes in this context-constrained PIN are calculated. Higher centrality for a gene indicates that the gene is more important to this network and should be more tightly related to breast cancer. Graph centrality of each gene in the context-constrained PIN is calculated and provided in Additional File 1. In a recent similar study performed by Chen et.al, based on a context-constrained network that obtained from literatures, 10 published gene signatures are integrated and a 54-genes signature is obtained [15]. This result is named as "Chen's signature" in the rest of this paper. For comparison, we also select 54 most important genes identified by each centrality definitions to construct gene signatures. Full list of genes in these graph centrality based signatures are provided in Table 2.

Since all the six graph centrality definitions are designed to measure the importance of a node to a graph, gene signatures identified by the six centrality measurements are similar with each other. A extremely case is all the top 54 genes identified by $E C$ and $S C$ are the same. By contrast, overlap between our results and Chen's gene signature is only $5-8(9.3 \%-14.8 \%$, p-value $<0.05)$.

An interesting result is found in our work. No matter which centrality measurement is used to evaluate the importance of genes in the context-constrained network, TP53 gene, which is already known as a tumor suppressor, is always the most important gene. Another similar example is breast cancer type 1 susceptibility protein (BRCA1). As we expected, our result also shows that BRCA1 plays an important role in the breast cancer. Other similar examples include epidermal growth factor receptor (EGFR), E1A binding protein p300(E300), Androgen receptor gene(AR) and so on (see Table 2).

However, another well-known breast cancer gene, BRCA2, is not included in any signature identified by the six centrality measurement. This is because BRCA2 is included in only one original signature and was discarded when we constructed the context-constrained PIN. On the one hand, the absence of BRCA2 is also a evidence to prove that the quality of existing breast cancer gene signatures is low, and on the other hand, the absence of BRCA2 in out signature indicates that our method can be improved by refining the signature genes collection method.

\section{Relationship among genes in gene signatures}

To investigate the relationship among genes in the gene signatures, seven sub PINs are constructed by projecting genes in each gene signature to the complete human

Table 1 Overlap among 94 breast cancer gene signatures

\begin{tabular}{|c|c|c|c|c|c|c|c|c|c|c|c|c|c|c|c|c|}
\hline Frequency & 1 & 2 & 3 & 4 & 5 & 6 & 7 & 8 & 9 & 10 & 11 & 12 & 14 & 15 & 16 & 17 \\
\hline Num of genes & 4143 & 1608 & 687 & 323 & 148 & 56 & 40 & 23 & 15 & 13 & 4 & 3 & 1 & 1 & 1 & 1 \\
\hline
\end{tabular}

There is very small overlap among distinct breast cancer signatures. Most genes exist in only one signature, and only 24 genes are included in 10 or more signatures. 
Table 2 Graph centrality based breast cancer signatures

\begin{tabular}{|c|c|c|c|c|c|}
\hline BC & $\mathrm{CC}$ & DC & EC & IC & SC \\
\hline TP53 & TP53 & TP53 & TP53 & TP53 & TP53 \\
\hline EGFR & ESR1 & EGFR & ESR1 & EGFR & ESR1 \\
\hline CTNNB1 & $A R$ & EP300 & EP300 & EP300 & EP300 \\
\hline SMAD3 & EGFR & ESR1 & $A R$ & ESR1 & AR \\
\hline ESR1 & EP300 & BRCA1 & BRCA1 & BRCA1 & BRCA1 \\
\hline EP300 & SMAD3 & CREBBP & CREBBP & CREBBP & CREBBP \\
\hline SRC & BRCA1 & SMAD3 & SMAD3 & AR & SMAD3 \\
\hline BRCA1 & CTNNB1 & AR & EGFR & SMAD3 & EGFR \\
\hline CREBBP & SRC & CTNNB1 & HDAC1 & SRC & HDAC1 \\
\hline$A R$ & CREBBP & SRC & STAT3 & CTNNB1 & STAT3 \\
\hline UBE2I & AKT1 & $\mathrm{HDAC1}$ & RB1 & HDAC1 & RB1 \\
\hline DYNLL1 & HDAC1 & CASP3 & CTNNB1 & RB1 & CTNNB1 \\
\hline CASP3 & STAT3 & $\mathrm{RB} 1$ & JUN & PIK3R1 & JUN \\
\hline ACTB & STAT1 & PIK3R1 & SRC & CASP3 & SRC \\
\hline AKT1 & XRCC6 & STAT3 & AKT1 & STAT3 & AKT1 \\
\hline HDAC1 & RB1 & UBE2I & SMARCA4 & AKT1 & SMARCA4 \\
\hline PIK3R1 & PIK3R1 & AKT1 & CDKN1A & SHC1 & CDKN1A \\
\hline ZBTB16 & PML & CDK2 & PML & CDK2 & PML \\
\hline ACVR1 & UBE2I & PCNA & STAT1 & JUN & STAT1 \\
\hline RB1 & HSPA8 & $\mathrm{SHC1}$ & CDK2 & STAT1 & CDK2 \\
\hline STAT1 & CASP3 & JUN & NCOA6 & UBE2I & NCOA6 \\
\hline HGS & CDK2 & DYNLL1 & HDAC2 & CDKN1A & HDAC2 \\
\hline CDK2 & SMARCA4 & STAT1 & PIK3R1 & PCNA & PIK3R1 \\
\hline YWHAZ & CDKN1A & ACTB & UBE2I & HDAC2 & UBE2I \\
\hline BCL2 & JUN & CDKN1A & XRCC6 & SMARCA4 & XRCC6 \\
\hline XRCC6 & $\mathrm{CDH} 1$ & MAPK14 & HIF1A & NFKB1 & HIF1A \\
\hline STAT3 & YWHAZ & YWHAZ & NFKB1 & $\mathrm{CDH} 1$ & NFKB1 \\
\hline PLK1 & MAPK14 & BCL2 & CASP3 & ACTB & CASP3 \\
\hline PCNA & PRKDC & HDAC2 & E2F1 & MAPK14 & E2F1 \\
\hline HSPA8 & HIF1A & ATM & CEBPB & LYN & CEBPB \\
\hline FN1 & SHC1 & LYN & PRKDC & XRCC6 & PRKDC \\
\hline VIM & STUB1 & NFKB1 & $\mathrm{SHC1}$ & ATM & SHC1 \\
\hline CD44 & HDAC2 & XRCC6 & NCOA2 & ZBTB16 & $\mathrm{NCOA} 2$ \\
\hline MAPK14 & ERBB2 & $\mathrm{CDH} 1$ & CCND1 & E2F1 & CCND1 \\
\hline GNB2L1 & HSPA4 & SMARCA4 & PCNA & YWHAZ & PCNA \\
\hline FANCA & BCL2 & EZR & TDG & BCL2 & TDG \\
\hline EEF1A1 & ZBTB16 & HGS & ERBB2 & PML & ERBB2 \\
\hline SKIL & LYN & ZBTB16 & HSPA4 & JAK1 & HSPA4 \\
\hline SHC1 & NCOA6 & PLK1 & ZBTB16 & EZR & ZBTB16 \\
\hline USP7 & PAK1 & CAV1 & RXRA & PRKDC & RXRA \\
\hline CAV1 & MDM4 & $\mathrm{E} 2 \mathrm{~F} 1$ & MAPK14 & JAK2 & MAPK14 \\
\hline JUN & CASP8 & JAK1 & JAK2 & CASP8 & JAK2 \\
\hline RXRA & CEBPB & RXRA & STUB1 & RXRA & STUB1 \\
\hline LYN & NFKB1 & FN1 & CDK7 & ERBB2 & CDK7 \\
\hline CDKN1A & JAK2 & HSPA8 & PTPN6 & HIF1A & PTPN6 \\
\hline HIF1A & PTPN6 & VAV1 & ATM & CAV1 & ATM \\
\hline ATM & HGS & ACVR1 & BCL3 & HSPA8 & $\mathrm{BCL} 3$ \\
\hline EZR & $E Z R$ & CASP8 & DDX5 & VAV1 & DDX5 \\
\hline PPP1CA & CAV1 & HIF1A & RBL1 & CDKN2A & RBL1 \\
\hline PAK1 & PIAS4 & JAK2 & FOS & NCOA6 & FOS \\
\hline PLSCR1 & TDG & PML & $\mathrm{CDH} 1$ & PTPN6 & $\mathrm{CDH} 1$ \\
\hline
\end{tabular}

Table 2 Graph centrality based breast cancer signatures (Continued)

\begin{tabular}{rrrrrr}
\hline TGFBR2 & IGF1R & CDKN2A & MDM4 & DYNLL1 & MDM4 \\
AURKA & DDX5 & EEF1A1 & ING1 & HGS & ING1 \\
KPNB1 & MET & ERBB2 & SIN3A & SIN3A & SIN3A \\
\hline
\end{tabular}

54 genes with highest graph centrality in the context-constrained PIN are selected to consist new breast cancer gene signatures. The genes in the six signatures identified by six graph centrality measurements are listed in this table.

PIN. As shown in Table 3, the sub PINs consisted by the genes in the graph centrality based gene signatures are much denser than that consisted by the genes in Chen's signature. We also can observe the significant difference in Figure 2. The most dense networks are consisted by the disease genes that are identified by $E C$ and $S C$.

\section{KEGG pathways enrichment analysis}

As shown in Table 4, the most significant KEGG pathway of all six graph centrality-based gene signatures are "pathways in cancer", but that of Chen's gene signature is "Cell cycle". Chen's gene signature is also annotated by "pathways in cancer", but the $p$-score is very low. It is obvious that graph centrality based method is more powerful than SPAN to identify cancer related genes in a constrained PIN.

\section{GO enrichment analysis}

Biological process GO enrichment analysis is performed on each gene signatures (see Table 5). For each signature, the GO biological process with highest p-score and corresponding p-score are presented in Table 5. Unlike the genes identified in Chen's study [15], which is annotated by term "cell cycle", five centrality-based gene signatures are annotated by "positive regulation of macromolecule metabolic process" and only the gene signature identified by $B C$ is annotated by "response to organic substance".

Table 3 Topological size of context-constrained PINs.

\begin{tabular}{ccc}
\hline & Proteins & Interactions \\
\hline BC & 54 & 238 \\
CC & 54 & 330 \\
DC & 54 & 279 \\
EC & 54 & 352 \\
IC & 54 & 312 \\
SC & 54 & 352 \\
Chen & $54(35)$ & 70 \\
\hline
\end{tabular}

To investigate the differences between graph centrality based gene signatures and that reported in previous literature, topological size of sub-network consisted by the genes in these gene signatures are calculated and presented in this table. It should be note that the sub-network consisted by the genes included in Chen's gene signature is not a connected graph. The number in parenthesis is the number of proteins of its biggest connected component. 


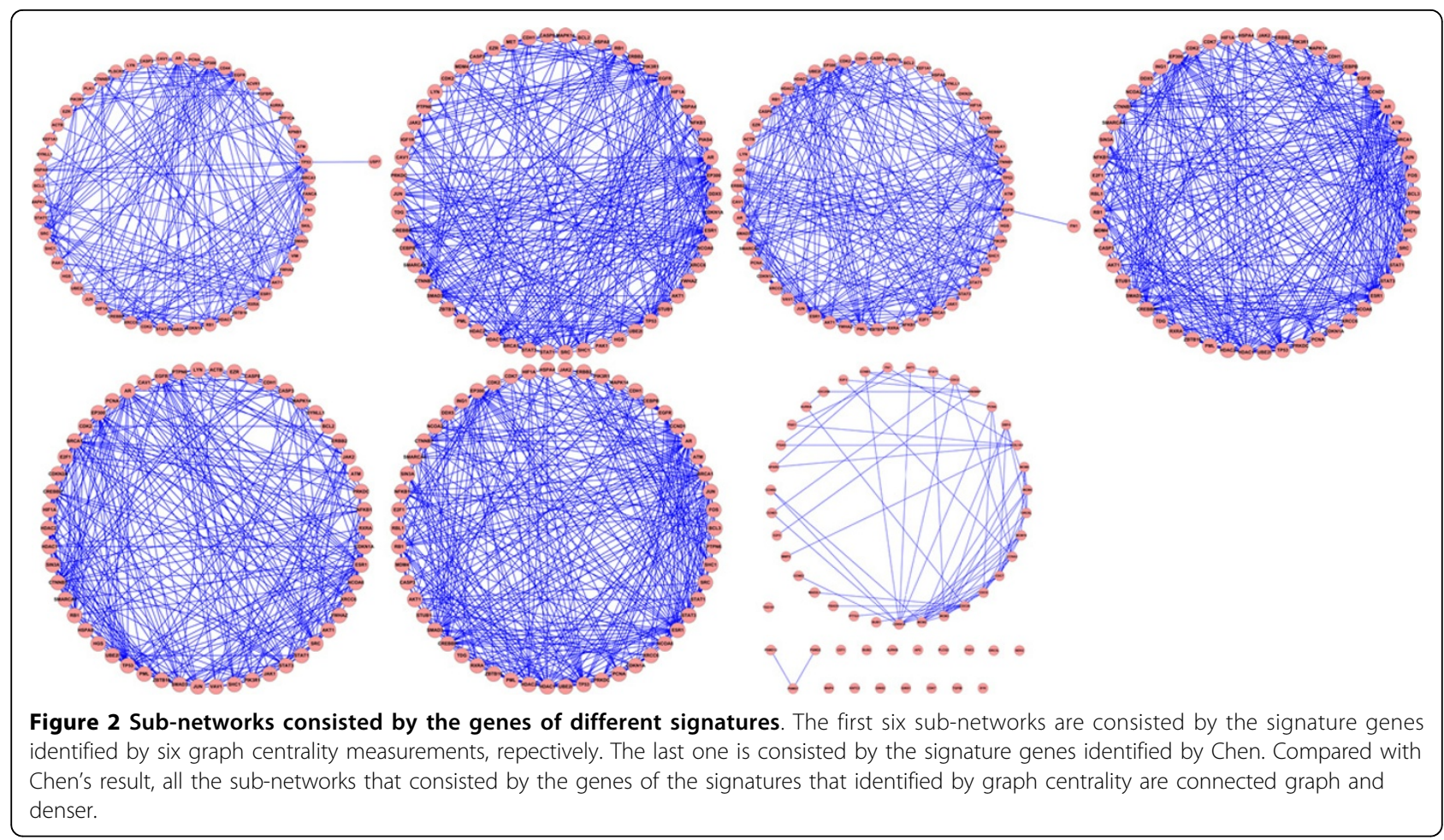

The structure of GO terms is a tree-like structure (see Figure 3. The term "regulation of biological regulation", which is located at the same level of "cell cycle", is the ancestor of "positive regulation of macromolecule metabolic process". According to G2SBC database [33], only 190 breast cancer genes are annotated by "Cell Cycle", but 1159 breast cancer genes are annotated by "regulation of biological process". This indicates that the gene signatures identified by graph centrality are probably more tightly related to breast cancer.

According to the $p$-score, both the two types of enrichment analysis suggested that $E C$ and $S C$ are probably the best choices to identify disease genes from context-constrained network and integrate different gene signatures. As reported in literatures, $S C$ also has superior performance in the discovery of essential proteins in protein interaction network [34] and essential proteins tend to have higher correlation with dominant and recessive mutants of disease genes [35]. This is possibly the reason that $S C$ and $E C$ outperformed other centrality measurements. We also note that the PINs that are consisted by the disease genes identified by $E C$ and $S C$ are denser than that of other centrality measurements (see Table 3). This indicates that breast cancer genes have tight and complicated relationship with each other.

\section{Validate of the prognostic potential of the subgraph centrality based gene signature}

Since $S C$ and $E C$ outperform in functional enrichment analysis, we tested the $S C$ and $E C$ based gene signatures in a genome-wide microarray dataset: GSE7390 [36]. This microarray dataset is downloaded from NCBI GEO

Table 4 KEGG pathways enrichment analysis results.

\begin{tabular}{cllll}
\hline Method & KEGG Pathway & Description & Annotated Genes & Corrected $\boldsymbol{p}$-score \\
\hline BC & hsa05200 & pathways in cancer & 23 & 13.09 \\
CC & hsa05200 & pathways in cancer & 29 & 18.92 \\
DC & hsa05200 & pathways in cancer & 31 & 21.59 \\
EC & hsa05200 & pathways in cancer & 28 & 25.74 \\
IC & hsa05200 & pathways in cancer & 30 & 20.22 \\
SC & hsa05200 & pathways in cancer & 28 & 25.74 \\
Chen & hsa04110 & Cell cycle & 25 & 26.80 \\
Chen & hsa05200 & pathways in cancer & 11 & 2.59 \\
\hline
\end{tabular}

To investigate the relationship between the gene signatures and cancer-related pathways, KEGG pathway enrichment analysis is preformed on these signatures. 
Table 5 Gene Ontology enrichment analysis results.

\begin{tabular}{cllc}
\hline Method & GO Term & Description & p-score \\
\hline BC & GO:0010033 & response to organic substance & 21.44 \\
CC & GO:0010604 & positive regulation of macromolecule metabolic process & 21.44 \\
DC & GO:0010604 & positive regulation of macromolecule metabolic process & 17.50 \\
EC & GO:0010604 & positive regulation of macromolecule metabolic process & 25.74 \\
IC & GO:0010033 & response to organic substance & 19.60 \\
SC & GO:0010604 & positive regulation of macromolecule metabolic process & 25.74 \\
Chen & GO:0007049 & cell cycle & 19.94 \\
\hline
\end{tabular}

To investigate the relationship between the gene signatures and cancer-related biological processes, Gene Ontology enrichment analysis is preformed on these signatures.

database and includes 198 breast cancer patients width different pre-diagnosed pathologic parameters.

We analyze the relationship between the $S C$ based signature genes and hormone receptor status using hierarchical clustering. The 198 patients in GSE7390 were divided into two main clusters (Additional File 2), the first cluster includes 118 samples, and the second cluster includes 80 samples. The mean value of each attribute of the samples in each cluster are listed in Table 6. As shown in Table 6, the tumor size and NPI Score of the

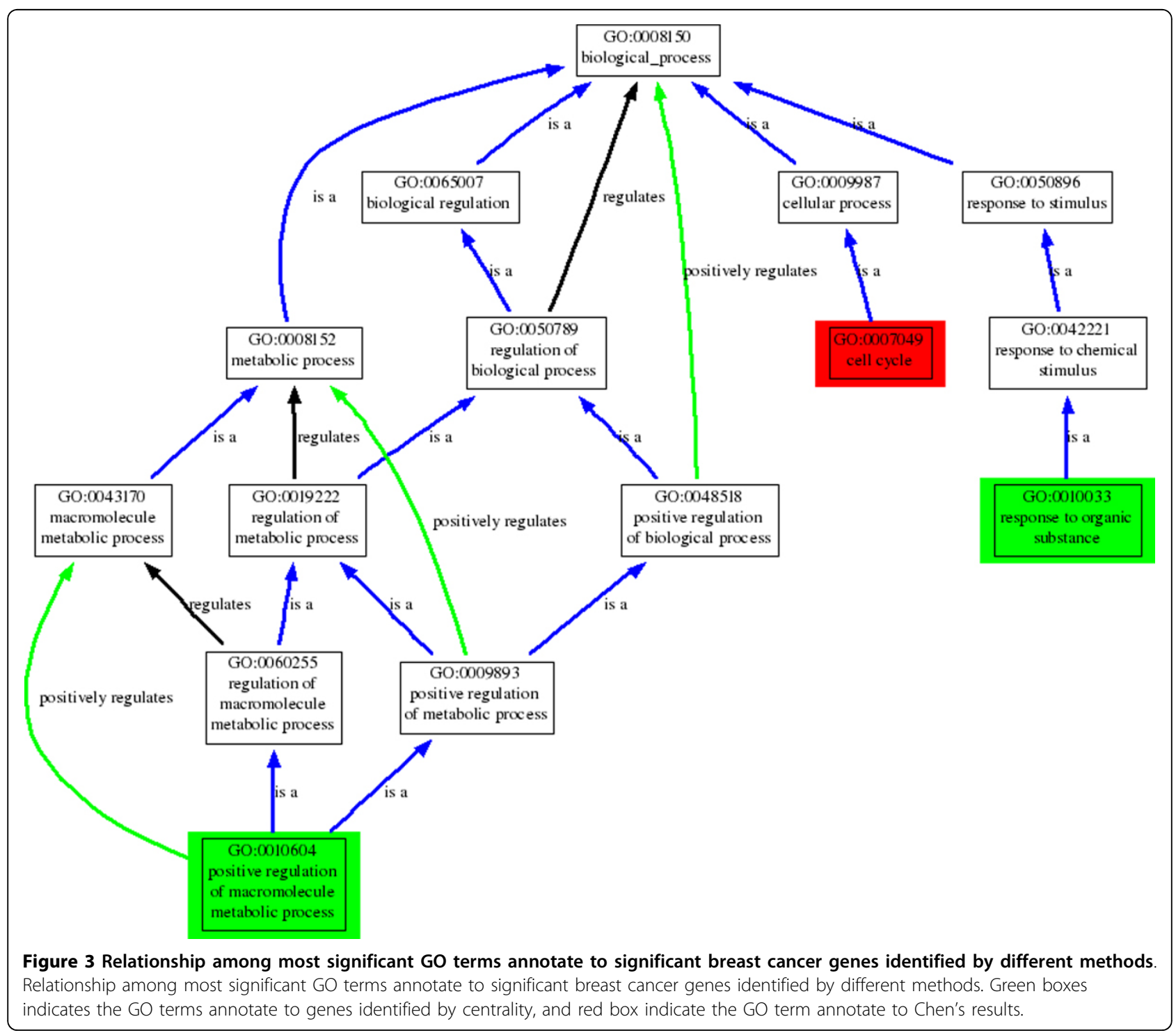


Table 6 Clinical outcome of two main clusters

\begin{tabular}{rrr}
\hline & $\begin{array}{r}\text { 118 samples } \\
\text { cluster }\end{array}$ & $\begin{array}{r}\mathbf{8 0} \text { samples } \\
\text { cluster }\end{array}$ \\
\hline Patient Age & 47 & 45 \\
Tumor Size(mm) & 2.06 & 2.36 \\
Disease-free survival (days) & 3498 & 3252 \\
Overall survival (days) & 4391 & 3792 \\
Distant metastasis-free survival & 4148 & 3667 \\
(days) & & \\
Time to distant metastasis & 4148 & 3667 \\
(days) & & \\
NPI Score & 3.4 & 4.17 \\
10-year overall survival & 83.38 & 74.98 \\
probability & & \\
\hline
\end{tabular}

Mean value of each clinical outcome recorded in the microarray dataset of the samples in each cluster is presented in this table.

first cluster is smaller than those of the second cluster. Disease-free survival time, overall survival time, distant metastasis-free survival time, 10-year overall survival probability and time to distant metastasis of the patients in the first cluster is longer than those of the patients in the second cluster. In another word, the condition of patients in the first cluster is much better than the second cluster of patients.

In the first cluster of patients, 104 of them were ER+; and in the second cluster of patients, only 30 of them were ER+. The $p$-value calculated by ANOVA is $2.47 \times$ $10^{-13}$. We also explored the relationship between the signature genes and the time to distant metastasis. In the first cluster, the time to distant metastasis of 97 patients were longer than 2000 days; and in the second cluster, that number is only 55 . The $p$-value is 0.04254 . Normally, $p$-value that smaller than 0.05 means the result is statistic significant and is not generated by chance. Such small $p$-value of the clustering result indicates that the $S C$ based signature is able to predict the clinical outcome very well.

\section{Discussion and conclusion}

Identification of genes which play important roles in the development of cancer is a critical problem needed to solve in current research of various cancers. Gene expression signatures provide a way to find significant cancer genes in given groups of patients. Due to the low overlap between heterogeneous signatures, how to integrate them is becoming a serious problem. Fortunately, as shown in this paper, graph centralities, especially $E C$ and $S C$, are useful tools to integrate existing different cancer gene signatures.

As well-known, weighted protein interaction network can be constructed by integrating functional annotations, and centrality is also can be extended to weighted network easily [20]. More promising results should be found in the weighted protein interaction network.
Besides this, other topological parameters from graph theory may improve this method as well.

\section{Additional material}

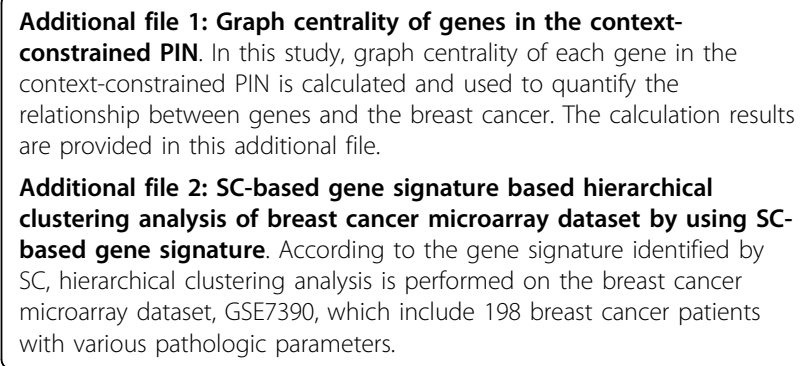

Additional file 2: SC-based gene signature based hierarchical clustering analysis of breast cancer microarray dataset by using SCbased gene signature. According to the gene signature identified by SC, hierarchical clustering analysis is performed on the breast cancer microarray dataset, GSE7390, which include 198 breast cancer patients with various pathologic parameters.

\section{Acknowledgements}

This work is supported in part by the National Natural Science Foundation of China under Grant No.61003124 and No.61073036, the Ph.D. Programs Foundation of Ministry of Education of China No.20090162120073, the Freedom Explore Program of Central South University No.201012200124, and the U.S. National Science Foundation under Grants CCF-0514750, CCF0646102, and CNS-0831634.

This article has been published as part of BMC Systems Biology Volume 5 Supplement 3, 2011: BIOCOMP 2010 - The 2010 International Conference on Bioinformatics \& Computational Biology: Systems Biology. The full contents of the supplement are available online at http://www.biomedcentral.com/ $1752-0509 / 5$ ? issue $=$ S3.

\section{Author details}

${ }^{1}$ School of Information Science and Engineering, Central South University, Changsha, 410083, China. ${ }^{2}$ Department of Computer Science, Georgia State University, Atlanta, GA30303, USA.

\section{Authors' contributions}

GC conceived and carried out this work under the guidance and supervision of JW. JW, GC and ML drafted the manuscript together. YP participated in revising the draft. All authors have read and approved the manuscript.

\section{Competing interests}

The authors declare that they have no competing interests.

Published: 23 December 2011

\section{References}

1. Itadani $\mathrm{H}$, Mizuarai $\mathrm{S}$, Kotani $\mathrm{H}$ : Can systems biology understand pathway activation? Gene expression signatures as surrogate markers for understanding the complexity of pathway activation. Curr Genomics 2008, 9(5):349-360.

2. van 't Veer $L$, Dai H, van de Vijver MJ, et al: Gene expression profiling predicts clinical outcome of breast cancer. Nature 2002, 415(6871):530-536.

3. van de Vijver MJ, He YD, van 't Veer $L$, et al: A Gene-Expression Signature as a Predictor of Survival in Breast Cancer. New England Journal of Medicine 2002, 347(25):1999-2009.

4. Chuang HY, Lee E, Liu YT, Lee D, Ideker T: Network-based classification of breast cancer metastasis. Mol Syst Biol 2007, 3:140.

5. Paik $S$, Shak $S$, Tang $G$, et al: A multigene assay to predict recurrence of tamoxifen-treated, node-negative breast cancer. N Engl J Med 2004, 351(27):2817-2826

6. Minn AJ, Gupta GP, Siegel PM, et al: Genes that mediate breast cancer metastasis to lung. Nature 2005, 436(7050):518-524.

7. Wang Y, Klijn JGM, Zhang Y, et al: Gene-expression profiles to predict distant metastasis of lymph-node-negative primary breast cancer. Lancet 2005, 365(9460):671-679. 
8. van Vliet MH, Reyal F, Horlings HM, et al: Pooling breast cancer datasets has a synergetic effect on classification performance and improves signature stability. BMC Genomics 2008, 9:375

9. Haibe-Kains B, Desmedt C, Piette F, Buyse M, Cardoso F, Veer LV, Piccart M, Bontempi G, Sotiriou C: Comparison of prognostic gene expression signatures for breast cancer. BMC Genomics 2008, 9:394.

10. Blazadonakis ME, Zervakis ME, Kafetzopoulos D: Complementary gene signature integration in multi-platform microarray experiments. IEEE Trans Inf Technol Biomed 2011, 15:155-163.

11. Lage K, Karlberg EO, Størling ZM, et al: A human phenome-interactome network of protein complexes implicated in genetic disorders. Nat Biotechnol 2007, 25(3):309-316.

12. Lee $Y$, Yang $X$, Huang $Y$, Fan $H$, et al: Network modeling identifies molecular functions targeted by miR-204 to suppress head and neck tumor metastasis. PLoS Comput Biol 2010, 6(4):e1000730.

13. Laere SV, der Auwera IV, den Eynden GGV, et al: Distinct molecular signature of inflammatory breast cancer by cDNA microarray analysis. Breast Cancer Res Treat 2005, 93(3):237-246.

14. Guo NL, Wan YW, Bose S, Denvir J, Kashon ML, Andrew ME: A novel network model identified a 13-gene lung cancer prognostic signature. Int J Comput Biol Drug Des 2011, 4:19-39.

15. Chen J, Sam L, Huang $Y$, et al: Protein interaction network underpins concordant prognosis among heterogeneous breast cancer signatures. Journal of Biomedical Informatics 2010, 43(3):385-396.

16. Chen JL, Li J, Stadler WM, Lussier YA: Protein-network modeling of prostate cancer gene signatures reveals essential pathways in disease recurrence. J Am Med Inform Assoc 2011, 18(4):392-402.

17. Culhane AC, Schwarzl T, Sultana R, et al: GeneSigDB-a curated database of gene expression signatures. Nucleic Acids Res 2010, , 38 Database: D716-D725.

18. Breitkreutz BJ, Stark C, Reguly T, et al: The BioGRID Interaction Database: 2008 update. Nucleic Acids Res 2008, 36 Database: D637-D640.

19. Prasad TSK, Goel R, Kandasamy K, et al: Human Protein Reference Database-2009 update. Nucleic Acids Res 2009, , 37 Database: D767-D772

20. Li M, Wang J, Wang H, Pan Y: Essential Proteins Discovery from Weighted Protein Interaction Networks. Bioinformatics Research and Applications 2010, 89-100.

21. Jeong H, Mason SP, Barabási AL, Oltvai ZN: Lethality and centrality in protein networks. Nature 2001, 411(6833):41-42.

22. Joy MP, Brock A, Ingber DE, Huang S: High-betweenness proteins in the yeast protein interaction network. J Biomed Biotechnol 2005 2005(2):96-103.

23. Wuchty S, Stadler PF: Centers of complex networks. J Theor Biol 2003, 223:45-53

24. Estrada E, Rodríguez-Velázquez JA: Subgraph centrality in complex networks. Phys Rev E Stat Nonlin Soft Matter Phys 2005, 71(5 Pt 2):056103.

25. Bonacich P: Power and Centrality: A Family of Measures. The American Journal of Sociology 1987, 92(5):1170-1182.

26. Stephenson K, Zelen M: Rethinking centrality: Methods and examples. Social Networks 1989, 11:1-37.

27. Hwang W, Cho Y, Zhang A, Ramanathan M: A novel functional module detection algorithm for protein-protein interaction networks. Algorithms Mol Biol 2006, 12:1-24.

28. Bu D: Topological structure analysis of the protein-protein interaction network in budding yeast. Nucleic Acids Res 2003, 31:2443-2450.

29. Li M, Chen Je, Wang JX, et al: Modifying the DPClus algorithm for identifying protein complexes based on new topological structures. BMC Bioinformatics 2008, 9:398.

30. Spirin $V$, Mirny L: Protein complexes and functional modules in molecular networks. PNAS 2003, 100:12123-12128.

31. Cho YR, Hwang W, Zhang A: Identification of Overlapping Functional Modules in Protein Interaction Networks: Information Flow-based Approach. ICDMW '06 Washington, DC, USA: IEEE Computer Society; 2006, 147-152.

32. Huang DW, Sherman BT, Lempicki RA: Systematic and integrative analysis of large gene lists using DAVID bioinformatics resources. Nat Protoc 2009, 4:44-57.

33. Mosca E, Alfieri R, Merelli l, et al: A multilevel data integration resource for breast cancer study. BMC Syst Biol 2010, 4:76.

34. Estrada E: Virtual identification of essential proteins within the protein interaction network of yeast. Proteomics 2006, 6:35-40.
35. Furney SJ, Albà MM, López-Bigas N: Differences in the evolutionary history of disease genes affected by dominant or recessive mutations. BMC Genomics 2006, 7:165.

36. Desmedt C, Piette F, Loi S, et al: Strong time dependence of the 76-gene prognostic signature for node-negative breast cancer patients in the TRANSBIG multicenter independent validation series. Clin Cancer Res 2007, 13(11):3207-3214

doi:10.1186/1752-0509-5-S3-S10

Cite this article as: Wang et al:: Integration of breast cancer gene signatures based on graph centrality. BMC Systems Biology 2011 5(Suppl 3):S10.

\section{Submit your next manuscript to BioMed Central and take full advantage of:}

- Convenient online submission

- Thorough peer review

- No space constraints or color figure charges

- Immediate publication on acceptance

- Inclusion in PubMed, CAS, Scopus and Google Scholar

- Research which is freely available for redistribution 